\title{
Gut microbial pathways for bile acid metabolism
}

\author{
Fabian Frost, Markus M. Lerch \\ Department of Medicine A, University Medicine Greifswald, Greifswald, Germany \\ Correspondence to: Markus M. Lerch, MD. Department of Medicine A, University Medicine Greifswald, Ferdinand-Sauerbruch-Straße, 17475 \\ Greifswald, Germany. Email: lerch@uni-greifswald.de. \\ Comment on: Funabashi M, Grove TL, Wang M, et al. A metabolic pathway for bile acid dehydroxylation by the gut microbiome. Nature \\ 2020;582:566-70.
}

Submitted Jan 11, 2021. Accepted for publication Feb 25, 2021.

doi: 10.21037/hbsn-21-11

View this article at: http://dx.doi.org/10.21037/hbsn-21-11

The human gut represents a complex ecosystem which harbours a diverse, predominantly anaerobic bacterial community. Intestinal microbiota are not just passive bystanders but active contributors to human metabolism interfering with numerous host metabolic pathways, which are necessary for the maintenance of human health. Considerations to promote human health by modifying the gut microbiota and their metabolic functions have boosted microbiome research in recent years. Despite current efforts, many microbial metabolic pathways such as the metabolism of bile acids by gut microbiota, which has first been described several decades ago, are still incompletely understood. A complete characterization of these pathways, will however, be necessary for attempts to manipulate gut microbial functions as a means of therapy.

Primary bile acids are synthesized in the liver from cholesterol mostly via a classical pathway which starts with $7 \alpha$-hydroxylation, catalyzed by cholesterol $7 \alpha$-hydroxylase or via an alternative pathway which begins with hydroxylation through sterol 27-hydroxylase (1). Through these pathways the liver primarily synthesizes chenodeoxycholic acid (CDCA) and cholic acid (CA), which are conjugated mostly with glycine or taurine before secretion into the bile ducts. Several of the enzymes necessary for the hepatic production of primary bile acids and their conjugation are regulated by gut microbiota (2). After secretion into the bile ducts, bile acids are utilized for the emulsification and absorption of lipids and fat-soluble vitamins. During their passage through the distal ileum, the vast majority of bile acids are reabsorbed by a sodiumdependent bile acid transporter (ASBT) and reach the liver via the portal circulation to be secreted again into the bile ducts, a process which has been termed enterohepatic circulation. A smaller proportion of bile acids, which undergo deconjugation by microbiota in the small bowel, are not reabsorbed by ASBT and pass into the colon where they are subject to microbial metabolism and modification into secondary bile acids (1).

The physiological function of bile acids goes beyond their role in lipid absorption and involves the regulation of gut microbiota directly or indirectly through modification of the host's immune response (3), and includes resistance against Clostridioides (C.) difficile infections (4). Moreover, primary bile acids or microbially modified secondary bile acids play a role in glucose homeostasis, insulin sensitivity and the development of fatty liver disease, the latter via the farnesoid X receptor (FXR) or G-protein-coupled receptor TGR5 $(1,5)$. These findings are especially noteworthy since obesity and its related phenotypes type 2 diabetes mellitus $(6,7)$ and fatty liver disease are themselves closely intertwined with gut microbiota changes that promote gut microbiome instability (8) and result in a dysbiosis impairing the microbiome's physiological contribution to host metabolism.

The impact of bile acids on gut microbiota structure and human metabolism via FXR or TGR5 signalling have made them an interesting target for the development of novel treatment approaches for either metabolic disease or to promote gut microbiota stability, e.g., to counter C. difficile infections. For such attempts to be successful, however, a deeper understanding of the enterohepatic cycle as well as the role of bile acid-metabolizing microbes in the enterohepatic circulation would be required.

A recent study by Funabashi et al. (9) significantly 
extends our understanding of how two of the most abundant secondary bile acids, deoxycholic acid (DCA) and lithocholic acid (LCA) (10), are synthesized by gut microbiota from primary bile acids involving $7 \alpha$-dehydroxylation. The microbial capability for $7 \alpha$-dehydroxylation is encoded in the bile-acid induced (bai) operon which contains eight genes (5). At first, the authors purified orthologues of each bai enzyme and demonstrated in vitro that six of these enzymes are needed to convert CA into DCA without requiring additional catalytic activity and they characterized the sequence of reactions in which each of the enzymes is involved. After determining the necessary set of enzymes for secondary bile acid synthesis, the authors further sought to create means for manipulating the ability of gut microbiota to perform this specific task. To this end, C. sporogenes were conjugated sequentially with three different plasmids together containing the bai operon and the resulting strain was then termed C. sporogenes MF001, which was able to convert CA to DCA and CDCA to LCA. When transferring C. sporogenes MF001 into germ-free mice the authors could demonstrate that these rodents, or more specifically their new gut microbiome, now had acquired the capability to convert dietary CA into fecal DCA whereas control mice did not.

The value of the work of Funabashi et al. is not only grounded in the thorough description of the microbial $7 \alpha$-dehydroxylation pathway and its branching points. The authors were also able to transfer the genes necessary for $7 \alpha$-dehydroxylation into C. sporogenes, giving the engineered strain the capability for synthesis of secondary bile acids. They thus demonstrated the potential for engineering microbial strains with the capability for synthesizing of specific metabolites. This technology can now be used to enhance the functional repertoire of probiotic formulations in order to treat not only metabolic disease. On the other hand, the engineered strain C. sporogenes MF001 was far less effective in converting CA to DCA compared to the native $7 \alpha$-dehydroxylating strain $C$. sindens. This shows that regulation of the bile acid $7 \alpha$-dehydroxylation pathway is clearly complex and involves additional regulatory genes that have neither been identified nor are they yet understood. The authors ought to be congratulated for advancing our understanding of the role of the microbiome in bile acid metabolism.

\section{Acknowledgments}

Funding: None.

\section{Footnote}

Provenance and Peer Review: This article was commissioned by the editorial office of Hepatobiliary Surgery and Nutrition. The article did not undergo external peer review.

Conflicts of Interest: Both authors have completed the ICMJE uniform disclosure form (available at https://hbsn. amegroups.com/article/view/10.21037/hbsn-21-11/coif). The authors have no conflicts of interest to declare.

Ethical Statement: The authors are accountable for all aspects of the work in ensuring that questions related to the accuracy or integrity of any part of the work are appropriately investigated and resolved.

Open Access Statement: This is an Open Access article distributed in accordance with the Creative Commons Attribution-NonCommercial-NoDerivs 4.0 International License (CC BY-NC-ND 4.0), which permits the noncommercial replication and distribution of the article with the strict proviso that no changes or edits are made and the original work is properly cited (including links to both the formal publication through the relevant DOI and the license). See: https://creativecommons.org/licenses/by-nc-nd/4.0/.

\section{References}

1. Wahlström A, Sayin SI, Marschall HU, et al. Intestinal Crosstalk between Bile Acids and Microbiota and Its Impact on Host Metabolism. Cell Metab 2016;24:41-50.

2. Sayin SI, Wahlström A, Felin J, et al. Gut microbiota regulates bile acid metabolism by reducing the levels of tauro-beta-muricholic acid, a naturally occurring FXR antagonist. Cell Metab 2013;17:225-35.

3. Campbell Y, Fantacone ML, Gombart AF. Regulation of antimicrobial peptide gene expression by nutrients and by-products of microbial metabolism. Eur J Nutr 2012;51:899-907.

4. Buffie CG, Bucci V, Stein RR, et al. Precision microbiome reconstitution restores bile acid mediated resistance to Clostridium difficile. Nature 2015;517:205-8.

5. Ridlon JM, Harris SC, Bhowmik S, et al. Consequences of bile salt biotransformations by intestinal bacteria. Gut Microbes 2016;7:22-39.

6. Thingholm LB, Rühlemann MC, Koch M, et al. Obese Individuals with and without Type 2 Diabetes Show Different Gut Microbial Functional Capacity and 
Composition. Cell Host Microbe 2019;26:252-64.e10.

7. Frost F, Kacprowski T, Rühlemann M, et al. Impaired Exocrine Pancreatic Function Associates With Changes in Intestinal Microbiota Composition and Diversity. Gastroenterology 2019;156:1010-5.

8. Frost F, Kacprowski T, Rühlemann M, et al. Long-term instability of the intestinal microbiome is associated with metabolic liver disease, low microbiota diversity, diabetes mellitus and impaired exocrine pancreatic function. Gut 2021;70:522-30.

9. Funabashi M, Grove TL, Wang M, et al. A metabolic pathway for bile acid dehydroxylation by the gut microbiome. Nature 2020;582:566-70.

10. Hamilton JP, Xie G, Raufman JP, et al. Human cecal bile acids: concentration and spectrum. Am J Physiol Gastrointest Liver Physiol 2007;293:G256-63.
Cite this article as: Frost F, Lerch MM. Gut microbial pathways for bile acid metabolism. HepatoBiliary Surg Nutr 2021;10(3):379-381. doi: 10.21037/hbsn-21-11 